\title{
High-order Discontinuous Galerkin Methods Applied to Multiphase Flows
}

\author{
Marc T. Henry de Frahan* \\ Mechanical Engineering Department, University of Michigan, Ann Arbor, MI 48109, USA \\ Loc Khieu ${ }^{\dagger}$ \\ Institute for Computational Science and Technology, Ho Chi Minh City, Vietnam \\ Eric Johnsen \\ Mechanical Engineering Department, University of Michigan, Ann Arbor, MI 48109, USA
}

\begin{abstract}
In this paper, we present an application of the interface-centered binary projection method to improve the order of accuracy of the discretization of the advection terms by the Discontinuous Galerkin method. We present our multi-gpu parallel implementation and perform weak and strong scaling of our code. We apply the dg method to the multifluid Euler equations. We present numerical simulations of two multiphase problems applicable to many engineering fields. Simulations of a shock interacting with a drop of water in air are compared to experimental data. We show good agreement between the simulations and the experiments. Finally, we investigate the dynamics of a supersonic water drop impacting a wall. Large negative pressures and high tensions inside the drop resulting from the impact can lead to possible cavitation erosion of the wall.
\end{abstract}

\section{Introduction}

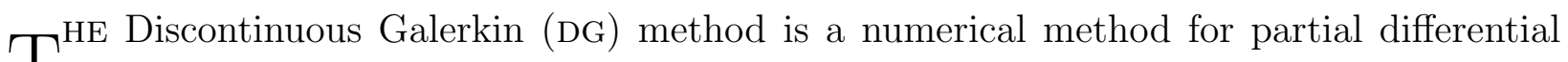
1 equations in which the solution is discretized in a computational cell through an expansion in terms of polynomial basis functions. This approach combines advantages of the finite element and the finite volume (FV) methods. In addition to being high-order accurate, the DG method is a compact-stencil scheme, so it is highly scalable on parallel architectures, and implementable on unstructured grids. The discontinuity in basis functions representing the solution naturally provides a means to introduce dissipation where needed. As with the FV methods, physical fluxes between neighboring cells are calculated using Riemann

\footnotetext{
*Graduate Student, University of Michigan, 1231 Beal Ave. 2043 Walter E. Lay Automotive Laboratory, Ann Arbor, MI 48109-2133

${ }^{\dagger}$ Researcher, Member AIAA

${ }_{\ddagger}^{\ddagger}$ Assistant Professor, University of Michigan, 1231 Beal Ave. 2043 Walter E. Lay Automotive Laboratory, Ann Arbor, MI 48109-2133
} 
solvers. The DG approach exhibits superconvergence properties for the advection terms, at a rate of $2 p+1$, where $p$ is the polynomial order. ${ }^{1,2}$ Cockburn and Shu popularized the Runge-Kutta Discontinuous Galerkin (RKDG)for time-dependent convection-dominated problems. ${ }^{3-7}$ Shocks and discontinuities can accurately be captured using limiters to dampen the oscillations that are caused by high-order interpolation across a discontinuity. ${ }^{4,8-13}$

Because of the discontinuous representation of the solution at the cell interfaces, the DG method is not naturally amenable to discretizing second order derivatives, which appear, for example, in the diffusion terms of the Navier-Stokes equations. Past approaches to resolving this issue have involved interior penalty methods for elliptic and parabolic equations ${ }^{14-16}$ and rewriting the second order terms as a system of first order derivatives. ${ }^{17,18}$ The drawback is that there is no consistent approach and the methods require tunable parameters. The Recovery discontinuous Galerkin (RDG) method was developed to provide a unified and consistent framework for discretizing second order derivatives. ${ }^{19-21}$ The method removes the discontinuity at the interface by recovering the underlying high-order polynomial spanning neighboring cells. This is done by matching polynomial moments in cells that share interfaces. The RDG method is stable, works in multiple dimensions, and can be applied to non-linear diffusion operators. It also exhibits superconvergent properties at a rate of $3 p+2$. However, using the RDG method leads to a mismatch in the convergence properties of the advection terms $(2 p+1)$ and the diffusion terms $(3 p+2)$. Improving the order of accuracy of the discretization of the advection term is therefore necessary.

Simulating interfaces in compressible multiphase flows is challenging because of large density and pressure ratios, as well as spurious oscillations due to discontinuities and numerical discretization. ${ }^{22-24}$ Our current approach involves interface capturing, in which, in analogy to shock capturing, the discontinuity is regularized over a few grid points. This approach is a logical framework for diffusion. It is conservative and avoids the reinitialization problems of interface tracking methods. We have developed a high-order accurate DG method for multiphase flows which prevents spurious pressure oscillations. ${ }^{13,25}$ Our present focus lies in the investigation of the dynamics of individual drops interacting with shocks and high speed flows. Our previous DG work combined with the improvements to the DG advection discretization will enable the investigations of high speed multiphase flows relevant to fuel injection problems, plasma deposition manufacturing, and turbomachinery. ${ }^{26,27}$

In Section II the present paper builds on previous research ${ }^{28}$ to fix the order of accuracy mismatch between the advection and diffusion discretizations by increasing the discretization order of accuracy of the advection terms. In Section IV we apply this method to the multifluid Euler equations to investigate the dynamics of a shock interacting with a water drop and a supersonic drop hitting a wall.

\section{Accuracy Improvements for the Advection Term of the Discontinuous Galerkin Method}

Systematic approaches to exploring improvements to the DG scheme have previously been presented. $^{28}$ This previous work has shown that an improved DG scheme for advection called interface-centered reconstruction with binary projection, denoted ICB, exhibits a $3 p+1$ order of accuracy and has reasonable stability properties. We recall the scheme here and apply it to the Euler equations. 
We consider without loss of generality the one-dimensional hyperbolic equation

$$
\frac{\partial u}{\partial t}+\frac{\partial F}{\partial x}=0
$$

for the conserved variable $u$, where $F(t, x, u)$ is the flux. In the DG scheme, the solution $u$ is expanded in each cell of the domain $\Omega_{j}$ in terms of $p+1$ basis functions $\phi_{n}(x), u_{j}(t, x) \approx$ $u_{h}(t, x)=\sum_{n=0}^{p} u_{n}(t) \phi_{n}(x)$. The flux at an arbitrary interface $j+1 / 2$ is evaluated using a Riemann solver which involves the left and right values of $u$ at that interface, $u_{L}$ and $u_{R}$, which are provided by the polynomial representation of $u_{j}$ and $u_{j+1}$. The cells $j-1, j$, and $j+1$ represent the computational stencil of the DG method used to evolve the solution in time. This compact stencil is one of the method's defining properties. We wish to keep the stencil compact when improving the DG method. Improvements to the basic DG advection scheme rely on increasing the polynomial order of the numerical representations $u_{j}$ and $u_{j+1}$ to calculate $\hat{u}_{j+1 / 2, L}(x)$ and $\hat{u}_{j+1 / 2, R}(x)$, from which $u_{L}$ and $u_{R}$ are evaluated.

The central idea of the ICB scheme is as follows. The reconstruction of $\hat{u}_{j+1 / 2, L}(x)$ is biased towards the left, cell $j$, meaning that the enhanced representation can contain more moments of $u_{j}$ than $u_{j+1}$,

$$
\begin{aligned}
& \int_{x_{j-1 / 2}}^{x_{j+1 / 2}} \phi_{n}^{j} \hat{u}_{j+1 / 2, L} \mathrm{~d} x=\int_{x_{j-1 / 2}}^{x_{j+1 / 2}} \phi_{n}^{j} u_{j} \mathrm{~d} x \quad n=0, \ldots, p \\
& \int_{x_{j+1 / 2}}^{x_{j+3 / 2}} \phi_{n}^{j+1} \hat{u}_{j+1 / 2, L} \mathrm{~d} x=\int_{x_{j+1 / 2}}^{x_{j+3 / 2}} \phi_{n}^{j+1} u_{j+1} \mathrm{~d} x \quad n \in \mathcal{N}
\end{aligned}
$$

where $\mathcal{N}$ is a subset of $\{0, \ldots, p\}$, the set of indexes of the moments of the original polynomial. A similar set of equations can be derived for $\hat{u}_{j+1 / 2, R}(x)$, which is then biased towards cell $j+1$. This binary reconstruction scheme achieves a $3 p+1$ order of accuracy if $\mathcal{N}$ contains $p$ original moments. This implies that there are $p+1$ subsets $\mathcal{N}$ formed with combinations of the set $\{0,1, \ldots, p\}$, leading to $p+1$ reconstructions schemes that exhibit $3 p+1$ accuracy. We denote the schemes of this type by $i c b \# 1[\# 2]$ where \#1 is the original polynomial order $p$ and $\# 2$ is the subset $\mathcal{N}$.

\section{II.A. Von Neumann Stability Analysis}

To study the stability of our DG enhancement scheme, we analyze the scalar linear advection equation 1 , where $F(t, x, u)=a u$ and $a>0$. The DG update equations are:

$$
\frac{\mathrm{d}}{\mathrm{d} t} \int_{\Omega_{j}} v_{j} u_{j} \mathrm{~d} x=-a \int_{\Omega_{j}} v_{j} \frac{\partial u_{j}}{\partial x} \mathrm{~d} x,
$$

and, using integration by parts,

$$
\frac{\mathrm{d}}{\mathrm{d} t} \int_{\Omega_{j}} v_{j} u_{j} \mathrm{~d} x=-a\left[v_{j} u_{j}\right]_{x_{j-1 / 2}}^{x_{j+1 / 2}}+a \int_{\Omega_{j}} \frac{\partial v_{j}}{\partial x} u_{j} \mathrm{~d} x,
$$

where $v_{j}$ is a test function in $\Omega_{j}$. By using the solution basis functions as the test functions, expanding $u(t, x)$ on the solution basis, and using an upwind flux (without loss of generality), we rewrite the update equations as a linear system for the solution coefficients:

$$
\frac{\Delta x}{a} \frac{\mathrm{d}}{\mathrm{d} t} \mathbf{u}_{j}=\mathbf{M}(T) \mathbf{u}_{j}
$$


where $T$ represents the translation of the solution by one cell: $T \mathbf{u}_{j}=\mathbf{u}_{j+1}$ and $T^{-1} \mathbf{u}_{j}=\mathbf{u}_{j-1}$. The Fourier transform of $\mathbf{M}(T), \widehat{\mathbf{M}}(\beta)$, is obtained by subsituting $\mathrm{T}$ for its transform $e^{i \beta}$. Calculations of the eigenvalues of $\widehat{\mathbf{M}}(\beta)$ and their respective Taylor-series expansions are used to compare with the exact differential operator, $\lambda_{e}=-i \beta$, and establish the stability and order of accuracy of the numerical scheme.

For $p=1$, we can construct two enhancement schemes, icb1[0] and icb1[0], to enhance the cell interface values. Von Neumann analysis of the resulting schemes showthat both schemes are stable $\left(\Re\left(\lambda_{i}\right) \leq 0 \forall i=0, \ldots, p\right)$, Figure 3 , and exhibit fourth order accuracy.

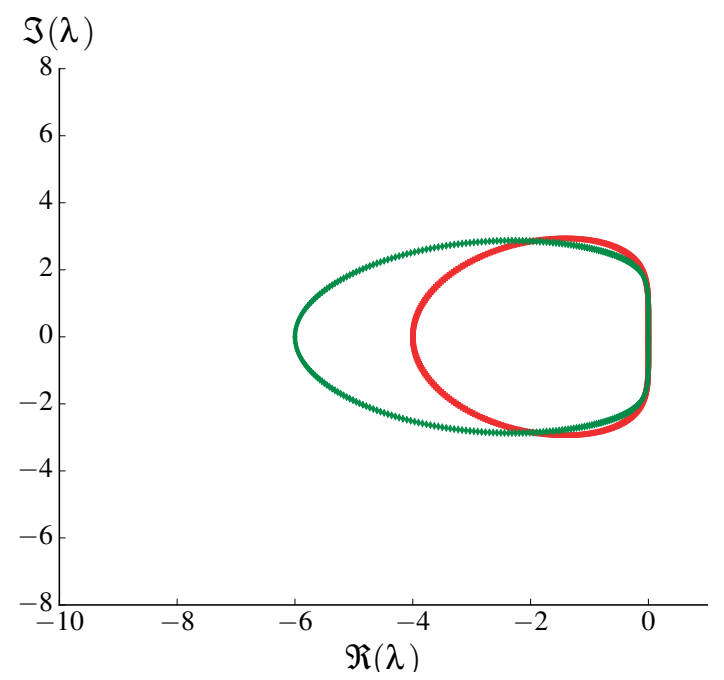

Figure 1: Loci of eigenvalues of the enhanced schemes for $p=1$ in the complex plane. Red squares: icb1[0]; green diamonds: icb1[0].

For $p=2$, there are three optimal enhancement schemes, icb2[0,1], icb2[1,2], and icb2 $[0,2]$. These schemes lead to seventh order accuracy (as opposed to fifth order accuracy for standard DG). However, as illustrated in Figure 2b, the real part of the eigenvalues are positive for some values of $\beta$. This incursion into the real positive half of the complex plane can lead to numerical instabilities if the time-marching scheme is inadequate. These incursion are small enough (their maximum value is 0.00073 ) to be covered by a standard Runge-Kutta 4 time integration scheme. However, this implies the existence of a lower bound on the CFL number to ensure that the scheme remains stable. For very small CFL numbers, these incursions will eventually lie outside the region of stability of the time-integration scheme.

For $p=2$, instead of using two moments from neighboring cells to enhance the interface, we can suboptimal sets consisting of using only one moment of the neighboring cell: icb2[0], icb2[1], and icb2[2]. These suboptimal schemes are stable and exhibit sixth order accuracy.

In conclusion, Von Neumann analysis of our enhanced shows that, for the one-dimensional scalar advection equation and for various suboptimal choices of the set $\mathcal{N}$, the scheme exhibits improved convergence and is stable. Using one moment from the neighboring cell for the enhancement scheme leads to a stable and $2 p+2$ order of accuracy scheme. We are currently working on stabilizing the icb schemes which use the optimal number of moments from the neighboring cells. 


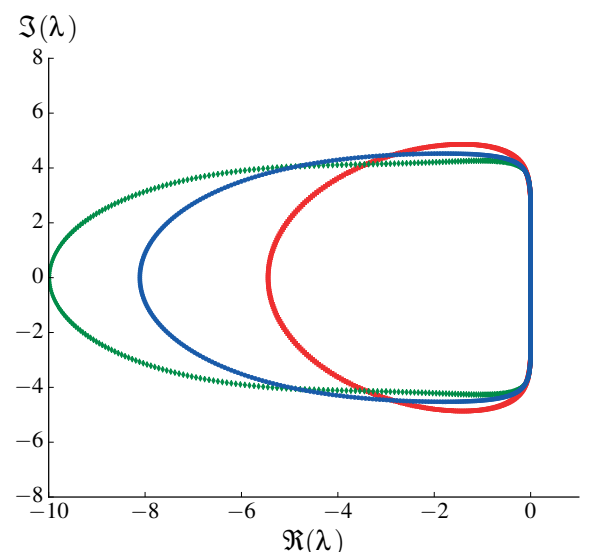

(a)

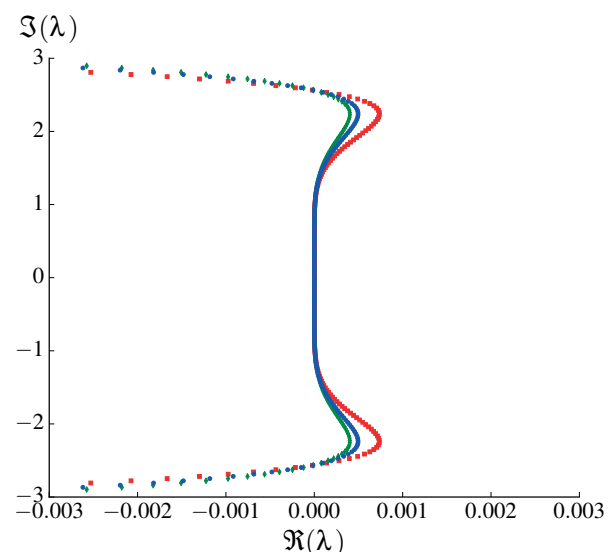

(b) Zoom of left figure

Figure 2: Loci of eigenvalues of the enhanced schemes for $p=2$ in the complex plane. Red squares: icb2[0,1]; green diamonds: icb2[1,2]; blue circles: icb2[1,2].

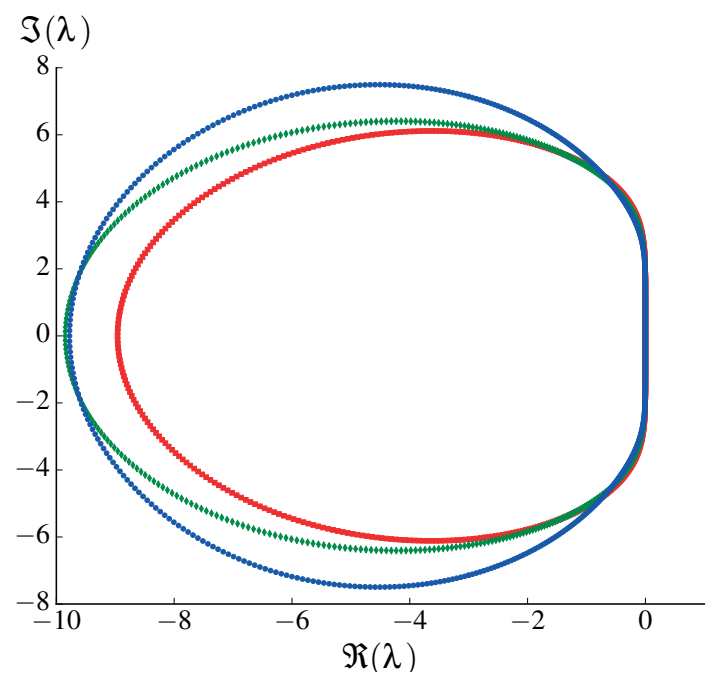

Figure 3: Loci of eigenvalues of the enhanced schemes for $p=2$ in the complex plane. Red squares: icb2[0]; green diamonds: icb2[1]; blue circles: icb2[2]. 


\section{Multi-GPU Implementation of the Discontinuous Galerkin Method}

Our highly parallel implementation of the DG method on graphics processing units (GPUs) uses the Message Passing Interface for multi-GPU parallelism to achieve high speedup. The domain is partitioned and the problem is initialized on each CPU. Each subdomain initialization is then sent to a GPU using the CUDA programming language. During the time integration, the GPUs perform the DG computations in their respective subdomains. At each time step of the time integration scheme the GPUs communicate their ghost cells to their host CPU, which then exchange ghost cell data with the other CPUs using MPI. This multi-step approach is robust and easily portable to many clusters.

Using the Extreme Science and Engineering Discovery Environment (XSEDE) ${ }^{29}$ and the Stampede cluster at the University of Texas, we perform weak and strong scaling analysis of our multi-GPU code. We consider a two-dimensional vortex advection problem on a periodic domain and use solution expansions using three basis functions $(p=2)$ in each direction.

The weak scaling study uses resolutions of $32^{2}, 64^{2}$, and $128^{2}$ cells per GPU, Figure 4a. The strong scaling study uses $256^{2}$ and $512^{2}$ cells in the domain, which corresponds to 589,824 and 2,359,296 total degrees of freedom, Figure 4b. The results scale well with increasing number of GPUs, especially if the significant increase in data movement is taken into account. In contrast to a single-GPU simulation, multi-GPU computations require data movement to and from the GPU and the host CPU and in between host CPUs. Current highperformance computing clusters such as Stampede only have one GPU per node. Because node-to-node communications are slow this significantly increases communication time.

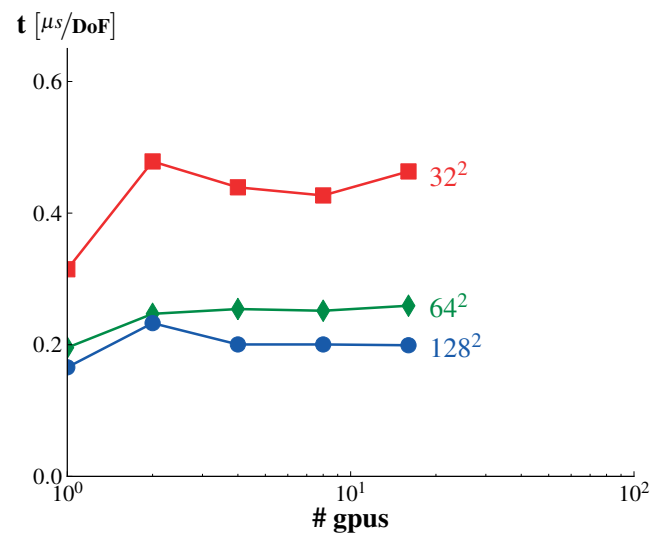

(a) Weak scaling

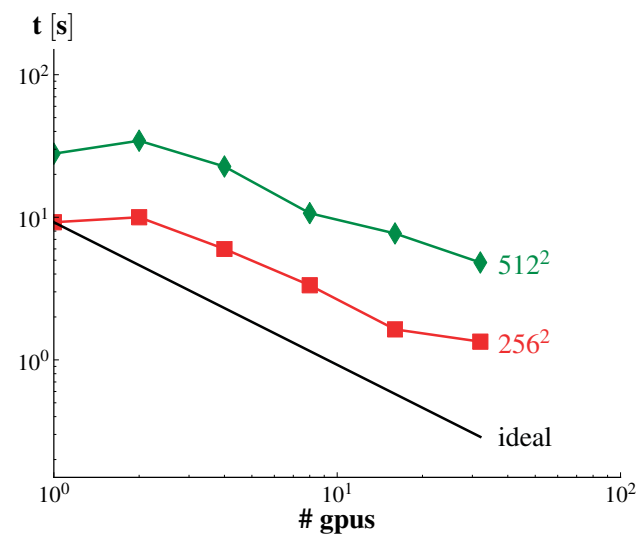

(b) Strong scaling.

Figure 4: Multi-GPU parallel scaling results using DG method for the two-dimensional vortex advection problem.

\section{Numerical Simulations of Multiphase Flows}

To simulate multiphase problems which are relevant to fuel injection, plasma deposition, raindrops impacting high speed vehicles, and turbomachinery, ${ }^{26,27}$ we solve the $2 \mathrm{D}$ multifluid 
Euler equations $(i, j=1,2)$ :

$$
\begin{aligned}
& \frac{\partial \rho}{\partial t}+\frac{\partial}{\partial x_{j}}\left(\rho u_{j}\right)=0, \\
& \frac{\partial\left(\rho u_{i}\right)}{\partial t}+\frac{\partial}{\partial x_{j}}\left(\rho u_{i} u_{j}+p \delta_{i j}\right)=0, \\
& \frac{\partial E}{\partial t}+\frac{\partial}{\partial x_{j}}\left[u_{j}(E+p)\right]=0,
\end{aligned}
$$

where $\rho$ is the density, $u_{i}$ is the velocity, $E=\rho e+\frac{1}{2} \rho u_{i} u_{i}$ is the total energy, $e$ is the internal energy, $p$ is the pressure and $\delta_{i j}$ is the Kronecker delta. The stiffened equation of state closes the system by relating the internal energy to the pressure

$$
\rho e=\frac{p+\gamma p_{\infty}}{\gamma-1}
$$

where $\gamma$ is the ratio of specific heats and $p_{\infty}$ is the stiffened constant. To avoid spurious pressure oscillations we solve two additional advection equations for the material properties

$$
\begin{aligned}
& \frac{\partial}{\partial t}\left(\frac{1}{\gamma-1}\right)+u_{j} \frac{\partial}{\partial x_{j}}\left(\frac{1}{\gamma-1}\right)=0, \\
& \frac{\partial}{\partial t}\left(\frac{\gamma p_{\infty}}{\gamma-1}\right)+u_{j} \frac{\partial}{\partial x_{j}}\left(\gamma \frac{p_{\infty}}{\gamma-1}\right)=0 .
\end{aligned}
$$

We use a third order Discontinuous Galerkin method to discretize these equations spatially. For the time integration we use the standard explicit fourth-order Runge-Kutta method with a Courant condition of 0.5. For all the problems in this paper we use the Roe Riemann solver and Lagrange basis functions. A modified hierarchical reconstruction procedure that prevents spurious pressure oscillations ${ }^{13}$ was used to limit the solution at shock and discontinuities. For the mesh generation and post-processing visualization we use Gmsh. ${ }^{30}$ Simulations of the multilayered Richtmyer-Meshkov and Rayleigh-Taylor instabilities $^{31}$ and experiments of blast-wave-driven shear flows in high-energy-density regimes ${ }^{32}$ have previously been performed with our code. In this section we will first investigate a strong shock impacting a drop of water in air and the subsequent drop deformation in the resulting supersonic flow. We then study a supersonic drop impacting a wall.

\section{IV.A. 2D Shock Drop Interaction}

In this problem, an initial 2D water drop of radius $r_{0}$ is stationary in air, Figure 5a. A March 2.5 shock coming from the left impinges on the drop. This setup is similar to past experiments and simulations of a shock interacting with a water column. ${ }^{33,34}$ The simulation domain size is $23 r_{0}$ in the $x$-direction and $16 r_{0}$ in the $y$-direction. The drop is initially located at $(x, y)=(0,0)$. The mesh is refined around the drop and the grid is stretched from $2 r_{0}$ above and below the centerline to the edge of the domain to allow for high resolution of the drop dynamics while maintaining a reasonable computational runtime. The entire domain contains approximately 5 million degrees of freedom. Each simulation was performed in parallel on eight GPUs for approximately 24 hours. The properties of the air are $\rho_{\text {air }}=$ $1.1765 \mathrm{~kg} / \mathrm{m}^{3}, \gamma_{\text {air }}=1.4$, and $p_{\infty, \text { air }}=0 \mathrm{~Pa}$. Those for water are $\rho_{\text {water }}=996 \mathrm{~kg} / \mathrm{m}^{3}, \gamma_{\text {water }}=5.5$, 
and $p_{\infty, \text { water }}=492115000 \mathrm{~Pa} .{ }^{35}$ All materials are initially at atmospheric pressure. The nondimensional time is defined as $t=T \frac{u_{s}}{2 r_{0}}$, where $T$ is the physical time and $u_{s}$ is the post-shock air velocity. Density is non-dimensionalized by $\rho_{\text {air }}$, velocity by $c_{\text {air }}$, and pressure by $\rho_{\text {air }} c_{\text {air }}^{2}$, where $c_{\text {air }}$ is the speed of sound in air.

As the shock impinges on the drop, it creates a supersonic flow around the drop. The initial shock is deflected by the drop and the reflected shock evolves into a bow shock, Figure 6a. Baroclinic vorticity generated at the drop surface by the passage of the shock creates a wake downstream of the drop which forms into a reentrant flow at later times, Figure $6 \mathrm{~b}$. This flow causes a decrease in the drop width and an increase in the height, Figure 6c. The strong coupling between the wave dynamics and the deforming geometry causes a series of compressions which steepen into shocks in the wake of the drop, Figures $6 \mathrm{c}$ and $6 \mathrm{~d}$.

We compare our simulation results to the experiments of the same setup ${ }^{33}$ in Figure 7 . There is good qualitative agreement with the experiments as the shape and wake of the drop in both the simulation and experiment look similar. The dark region upstream of the drop in the experimental pictures is most likely due to the experimental visualization technique which might be capturing evaporation effects or might be distorted by edge effects from the walls holding the water column. Experimental measurements of the drop width are accurately described by the simulation, Figure 8. There is very good agreement early in time and this agreement improves as the mesh is refined. Late time discrepancies can be explained by 3D effects and transport phenomena such as evaporation which are not modeled in the simulations.

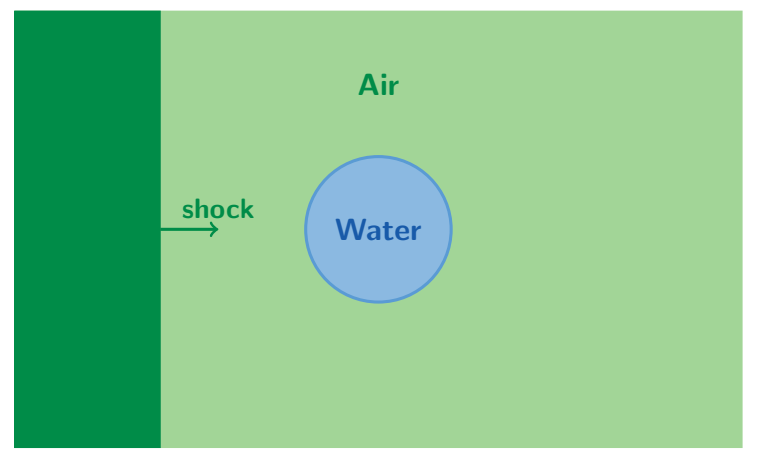

(a) Shock impinging on a drop of water in air.

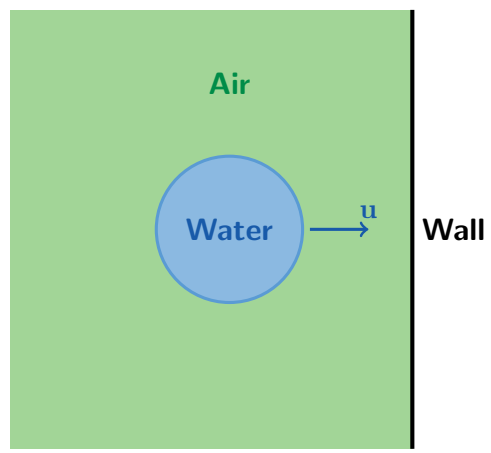

(b) Supersonic drop hitting a wall.

Figure 5: Problem setups for the multiphase flows.

\section{IV.B. Supersonic Drop Impact}

A drop located $4 r_{0}$ from a wall is moving at Mach 1.5 in initially quiescent air towards the wall, Figure 5b. The domain size is $13 r_{0}$ in the $x$-direction and $26 r_{0}$ in the $y$-direction. The mesh is refined around the bubble and along its trajectory towards the wall. Grid stretching is applied far from the drop and wall to minimize boundary effects and reduce computational cost. The non-dimensional time is defined as $t=T \frac{c_{\text {air }}}{r_{0}}$.

As the drop travels through the air it creates a bow shock. The box shock impinges on the wall and is reflected back towards the drop, Figure 9a. Soon after the drop hits the wall, generating a large pressure at the wall through the water hammer effect, Figure 9b. 


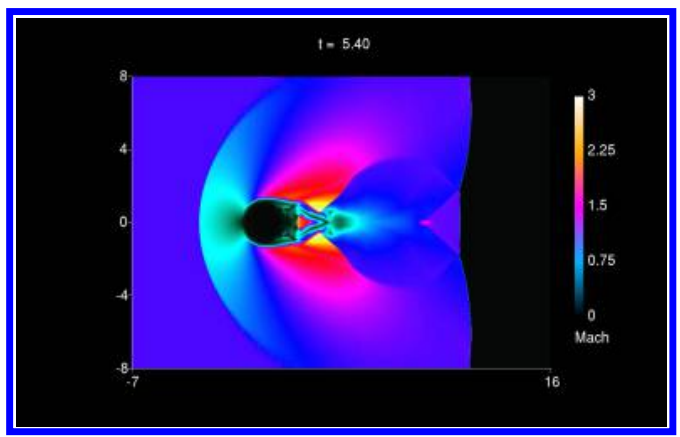

(a) $t=5.4$

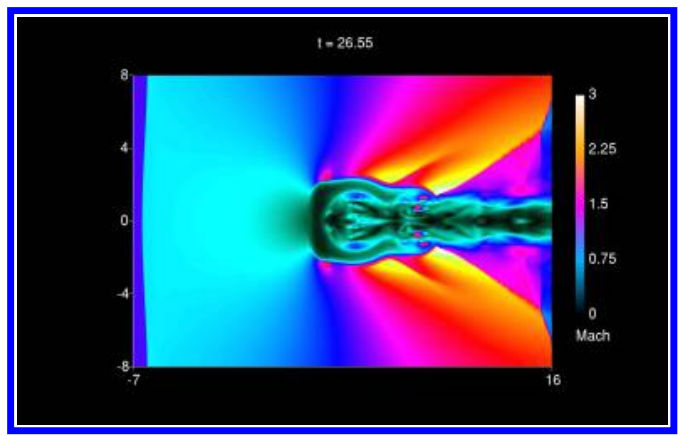

(c) $t=26.55$

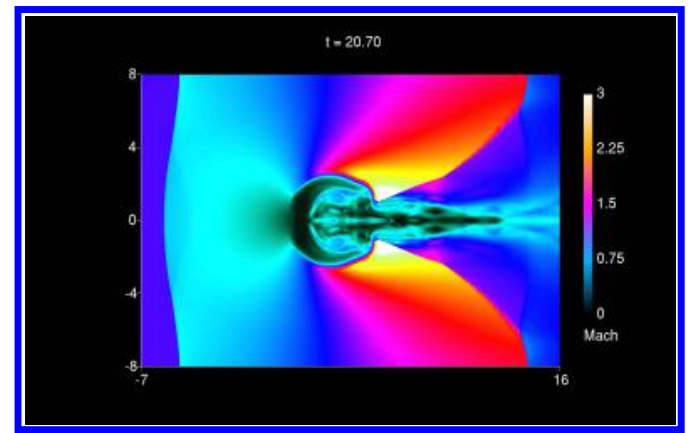

(b) $t=20.7$

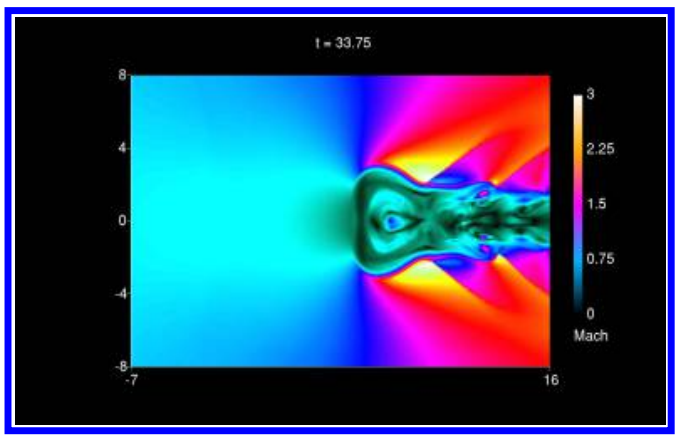

(d) $t=33.75$

Figure 6: Mach number contours for a 2.5 shock Mach number.

The shock from the wall traverses the drop and is reflected at the back of the drop as a rarefaction inside the drop, Figure 9c. This reflected rarefaction wave at the back of the drop causes a large pressure decrease and high tension inside the drop. Measurements of the pressure at the wall at three different location along the wall ( $h=0$ (drop centerline), $h=1 r_{0}$, and $h=2 r_{0}$ ) show the first pressure rise due to the bow shock, Figure 10. The large pressure jump due to water hammer effect when the drop hits the wall follows soon after. High negative wall pressures later in time are caused by the reflected rarefaction. These large tensions are a possible mechanism for cavitation in the drop and subsequent erosion of the wall.

\section{Conclusions}

In this paper, we recalled an improved advection scheme for the DG method. This improvement addresses the mismatch between the $3 p+1$ order of accuracy of the RDG method applied to diffusive terms and the $2 p+1$ order of accuracy of the standard advection DG method. We applied this novel scheme to the Euler equations. We developed a unique framework which enables the use of a superconvergent DG method with a consistent order of accuracy for both the diffusion and advection terms. Using the high-order DG method combined with a non-oscillatory reconstruction procedure, we solved two multiphase problems of relevance to many engineering applications. We showed very good qualitative and quantitative agreements between simulation results and past experiments. ${ }^{33}$ The reentrant flow in the wake of the drop caused by baroclinic vorticity deposition induces a decrease in the drop width and an increase in its height. Simulations results of a supersonic drop hitting a 


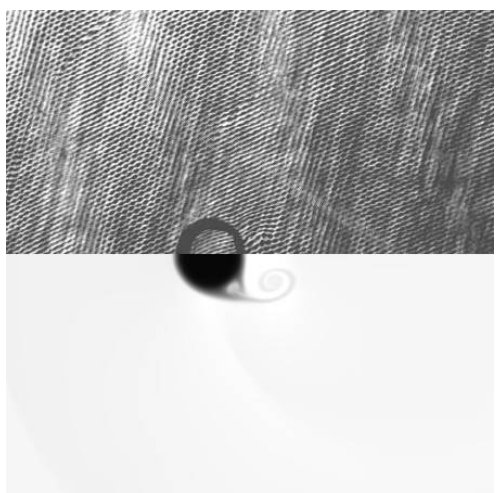

(a) $t=3.137$

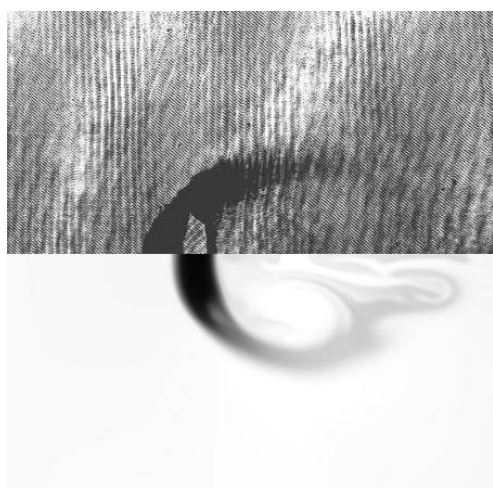

(d) $t=15.919$

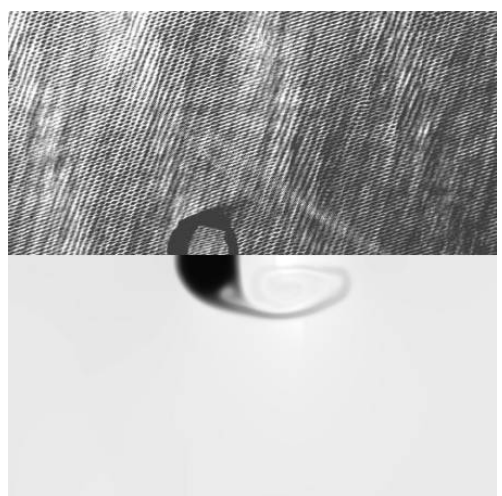

(b) $t=6.335$

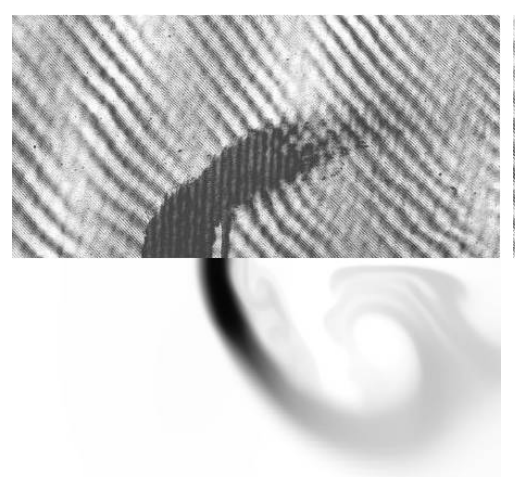

(e) $t=25.544$

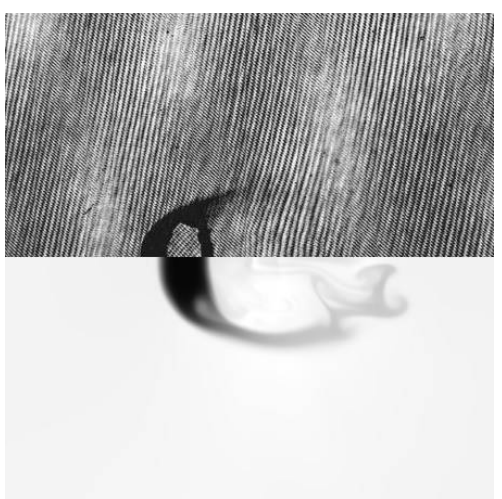

(c) $t=12.578$

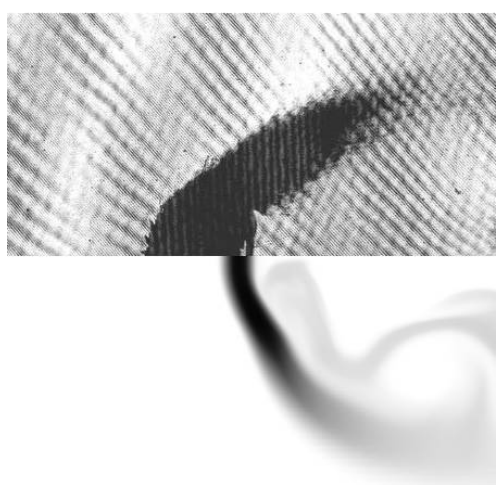

(f) $t=30.885$

Figure 7: Comparison of experimental results $^{33}$ (top) and simulated density (bottom) at a 1.18 shock Mach number.

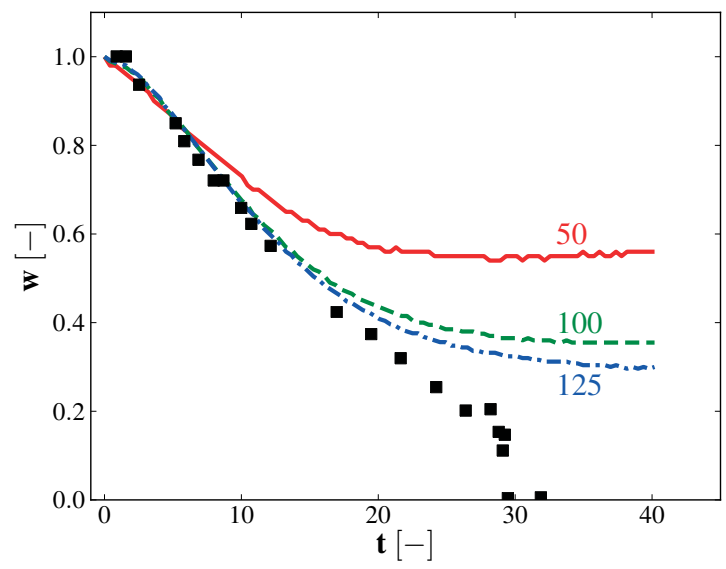

Figure 8: Comparison of the normalized drop width as a function of time between simulations and experiments at a 1.18 shock Mach number. Solid red: 50; dashed green: 100; dot-dashed blue: 125 cells per radius. Black squares: experimental results. ${ }^{33}$ 


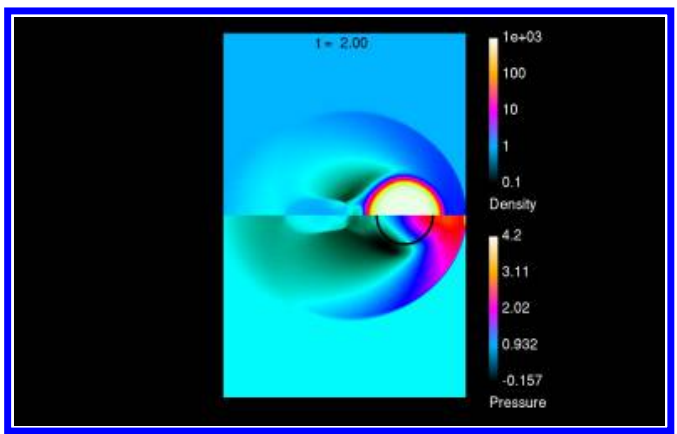

(a) $t=2$

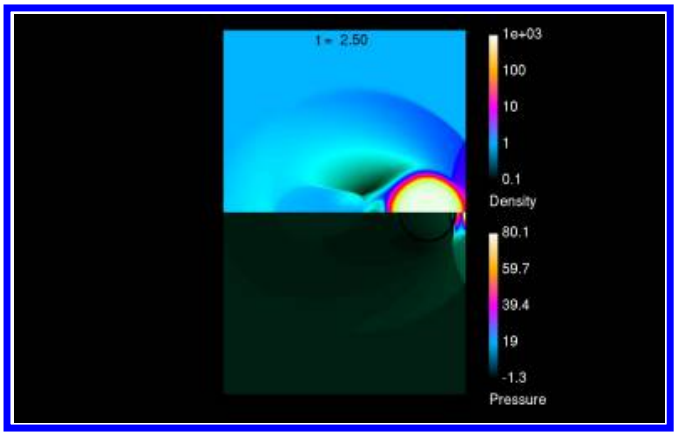

(b) $t=2.5$

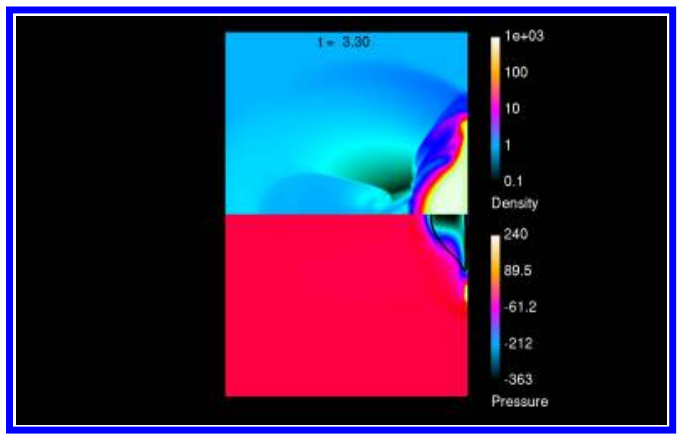

(c) $t=3.3$

Figure 9: Simulation results of non-dimensional density (top half) and pressure (bottom half) for a Mach 2.5 drop hitting a wall.

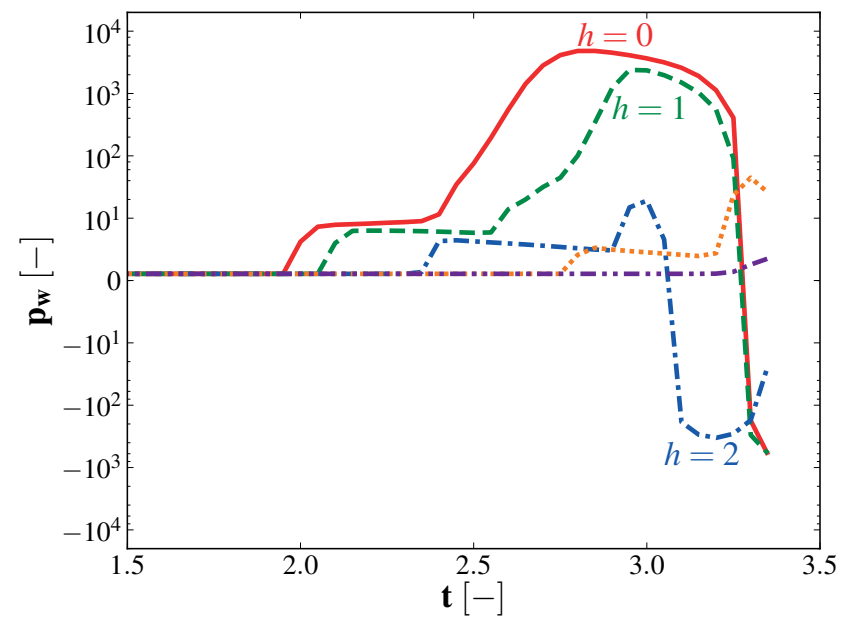

Figure 10: Pressure at the wall at three different locations. 
wall indicate that large negative pressures and high tensions could lead to cavitation erosion at the wall. For this latter problem, future work will include investigating the effect of the initial drop location on the wall pressure jump, bow shock interaction effects, and modeling air bubbles inside the drop.

\section{Acknowledgements}

This research was supported in part by the DOE/NNSA under the predictive Science Academic Alliance Program by Grant No. DEFC52-08NA28616, by ONR grant N00014-12-10751 under Dr. Ki-Han Kim, by NSF grant CBET 1253157, through computational resources and services provided by Advanced Research Computing at the University of Michigan, Ann Arbor, and used the Extreme Science and Engineering Discovery Environment (XSEDE), which is supported by National Science Foundation grant number ACI-1053575.

\section{References}

-1 Adjerid, S., Devine, K. D., Flaherty, J. E., and Krivodonova, L., "A posteriori error estimation for discontinuous Galerkin solutions of hyperbolic problems," Comput. Methods Appl. Mech. Engrg., Vol. 191, No. 11-12, 2002, pp. 1097-1112.

$\triangleright^{2}$ Adjerid, S. and Massey, T. C., "Superconvergence of discontinuous Galerkin solutions for a nonlinear scalar hyperbolic problem," Comput. Methods Appl. Mech. Engrg., Vol. 195, No. 25-28, May 2006, pp. 33313346.

${ }^{3}$ Cockburn, B., Lin, G., and Shu, C.-W., "TVB Runge-Kutta local projection discontinuous Galerkin finite element method for conservation laws III: One-dimensional systems," J. Comput. Phys., Vol. 84, No. 1, 1989, pp. 90-113.

${ }^{4}$ Cockburn, B. and Shu, C.-W., "TVB Runge-Kutta local projection discontinuous Galerkin finite element method for conservation laws II: General framework," Math. Comput., Vol. 52, No. 186, 1989, pp. 411-435.

${ }^{5}$ Cockburn, B., Hou, and Shu, C.-W., "The Runge-Kutta local projection discontinuous Galerkin finite element method for conservation laws IV: The multidimensional case," Math. Comput., Vol. 54, No. 190, 1990, pp. 545-581.

${ }^{6}$ Cockburn, B. and Shu, C.-W., "The local discontinuous Galerkin method for time-dependent convection-diffusion systems," SIAM J. Numer. Anal., Vol. 35, No. 6, 1997, pp. 2440-2463.

$\nabla^{7}$ Cockburn, B. and Shu, C.-W., "The Runge-Kutta discontinuous Galerkin method for conservation laws V: Multidimensional systems," J. Comput. Phys., Vol. 141, No. 2, 1997, pp. 199-224.

${ }^{8}$ Biswas, R., Devine, K. D., and Flaherty, J. E., "Parallel, adaptive finite element methods for conservation laws," Appl. Numer. Math., Vol. 14, No. 1-3, April 1994, pp. 255-283.

$\checkmark{ }^{9}$ Krivodonova, L., "Limiters for high-order discontinuous Galerkin methods," J. Comput. Phys., Vol. 226, No. 1, Sept. 2007, pp. 879-896.

${ }^{10}$ Kuzmin, D., "A vertex-based hierarchical slope limiter for p-adaptive discontinuous Galerkin methods," J. Comput. Appl. Math., Vol. 233, No. 12, April 2010, pp. 3077-3085.

${ }^{11}$ Liu, Y., Shu, C.-W., Tadmor, E., and Zhang, M., "Central discontinuous Galerkin methods on overlapping cells with a nonoscillatory hierarchical reconstruction," SIAM J. Numer. Anal., Vol. 45, No. 6, 2007, pp. 2442.

${ }^{12} \mathrm{Xu}, \mathrm{Z}$., Liu, Y., and Shu, C.-W., "Hierarchical reconstruction for discontinuous Galerkin methods on unstructured grids with a WENO-type linear reconstruction and partial neighboring cells," J. Comput. Phys., Vol. 228, No. 6, April 2009, pp. 2194-2212.

${ }^{13}$ Henry de Frahan, M. T., Varadan, S., and Johnsen, E., "A new limiting procedure for discontinuous 
Galerkin methods applied to compressible multiphase flows with shocks and interfaces," J. Comput. Phys., Vol. 280, No. 0, 2015, pp. 489 - 509.

${ }^{14}$ Arnold, D. N., Brezzi, F., Cockburn, B., and Marini, L. D., "Unified analysis of discontinuous Galerkin methods for elliptic problems," SIAM Journal on Numerical Analysis, Vol. 39, No. 5, 2002, pp. 1749-1779.

$\checkmark{ }^{15}$ Brezzi, F., Manzini, G., Marini, D., Pietra, P., and Russo, A., "Discontinuous Galerkin approximations for elliptic problems," Numerical Methods for Partial Differential Equations, Vol. 16, No. 4, 2000, pp. 365378.

${ }^{16}$ Douglas, J. and Dupont, T., "Interior penalty procedures for elliptic and parabolic Galerkin methods," Computing methods in applied sciences, 1976, pp. 207-216.

$\checkmark{ }^{17}$ Bassi, F. and Rebay, S., "A High-Order Accurate Discontinuous Finite Element Method for the Numerical Solution of the Compressible NavierStokes Equations," J. Comput. Phys., Vol. 131, No. 2, 1997, pp. 267-279.

${ }^{18}$ Peraire, J. and Persson, P.-O., "The Compact Discontinuous Galerkin (CDG) Method for Elliptic Problems," SIAM J. Sci. Comput., Vol. 30, No. 4, 2007, pp. 25.

$\checkmark{ }^{19}$ Lo, M. and van Leer, B., "Recovery-Based Discontinuous Galerkin for Navier-Stokes Viscous Terms," AIAA paper, , No. June, 2011, pp. AIAA-2011-3406.

${ }^{20}$ van Leer, B. and Lo, M., "Unification of Discontinuous Galerkin Methods for Advection and Diffusion 1 Introduction : history of RDG," New Horizons, , No. January, 2009, pp. 1-12.

${ }^{21}$ van Leer, B. and Lo, M., "A Discontinuous Galerkin Method for Diffusion Based on Recovery," Fluid Dynamics, , No. June, 2007.

22 Johnsen, E. and Colonius, T., "Implementation of WENO schemes in compressible multicomponent flow problems," J. Comput. Phys., Vol. 219, No. 2, Dec. 2006, pp. 715-732.

23 Johnsen, E., "On the treatment of contact discontinuities using WENO schemes," J. Comput. Phys., Vol. 230, 2011, pp. 8665-8668.

$\checkmark{ }^{24}$ Movahed, P. and Johnsen, E., "A solution-adaptive method for efficient compressible multifluid simulations, with application to the Richtmyer-Meshkov instability," J. Comput. Phys., Vol. 239, 2013, pp. 166-186.

${ }^{25}$ Henry de Frahan, M. T. and Johnsen, E., "Discontinuous Galerkin method for multifluid Euler equations," 21st AIAA Computational Fluid Dynamics Conference, American Institute of Aeronautics and Astronautics, 2013.

$\checkmark{ }^{26}$ Haller, K. K., Ventikos, Y., Poulikakos, D., and Monkewitz, P., "Computational study of high-speed liquid droplet impact," J. Appl. Phys., Vol. 92, No. 5, 2002, pp. 2821-2828.

${ }^{27}$ Haller, K. K., Poulikakos, D., Ventikos, Y., and Monkewitz, P., "Shock wave formation in droplet impact on a rigid surface: lateral liquid motion and multiple wave structure in the contact line region," $J$. Fluid Mech., Vol. 490, 9 2003, pp. 1-14.

${ }^{28} \mathrm{Khieu}, \mathrm{L}$. and Johnsen, E., "Analysis of Improved Advection Schemes for Discontinuous Galerkin Methods," 2014.

-29 Towns, J., Cockerill, T., Dahan, M., Foster, I., Gaither, K., Grimshaw, A., Hazlewood, V., Lathrop, S., Lifka, D., Peterson, G. D., Roskies, R., Scott, J. R., and Wilkens-Diehr, N., "XSEDE: Accelerating Scientific Discovery," Computing in Science and Engineering, Vol. 16, No. 5, 2014, pp. 62-74.

${ }^{30}$ Geuzaine, C. and Remacle, J.-F., "Gmsh: A 3-D finite element mesh generator with built-in pre- and post-processing facilities," Int. J. Numer. Methods Eng., Vol. 79, No. 11, Sept. 2009, pp. 1309-1331.

${ }^{31}$ Henry de Frahan, M. T. and Johnsen, E., "Numerical simulations of a shock interacting with successive interfaces using the Discontinuous Galerkin method: the multilayered Richtmyer-Meshkov and RayleighTaylor instabilities," Shock Waves, 2014.

${ }^{32}$ Di Stefano, C. A., Malamud, G., Henry de Frahan, M. T., Kuranz, C. C., Shimony, A., Klein, S. R., Drake, R. P., Johnsen, E., Shvarts, D., Smalyuk, V. A., and Martinez, D., "Observation and modeling of mixing-layer development in high-energy-density, blast-wave-driven shear flow," Phys. Plasmas, Vol. 21, No. 5, May 2014, pp. 056306.

\$33 Igra, D. and Takayama, K., "Experimental Investigation of Two Cylindrical Water Columns Subjected to Planar Shock Wave Loading," J. Fluids Eng., Vol. 125, No. 2, 2003, pp. 325.

${ }^{34}$ Meng, J. and Colonius, T., "Droplet Breakup in High-Speed Gas Flows," 8th Int. Conf. Multiph. Flow, No. 2011, ICMF, Jeju, Korea, 2013.

13 of 14

American Institute of Aeronautics and Astronautics 
${ }^{35}$ Cocchi, J. P., Saurel, R., and Loraud, J. C., "Treatment of interface problems with Godunov-type schemes," Shock Waves, Vol. 5, No. 6, May 1996, pp. 347-357. 